University of Montana

ScholarWorks at University of Montana

$1-21-2015$

\title{
Postnatal Growth Rates Covary Weakly with Embryonic Development Rates and Do Not Explain Adult Mortality Probability among Songbirds on Four Continents
}

\author{
Thomas E. Martin \\ University of Montana - Missoula \\ Oteyza C. Oteyza \\ University of Montana - Missoula \\ Adam E. Mitchell \\ University of Montana - Missoula \\ Ahva L. Potticary \\ University of Montana - Missoula \\ Penn Lloyd \\ University of Cape Town
}

Follow this and additional works at: https://scholarworks.umt.edu/wildbio_pubs

Part of the Poultry or Avian Science Commons

Let us know how access to this document benefits you.

\section{Recommended Citation}

Martin, Thomas E.; Oteyza, Oteyza C.; Mitchell, Adam E.; Potticary, Ahva L.; and Lloyd, Penn, "Postnatal Growth Rates Covary Weakly with Embryonic Development Rates and Do Not Explain Adult Mortality Probability among Songbirds on Four Continents" (2015). Wildlife Biology Faculty Publications. 94. https://scholarworks.umt.edu/wildbio_pubs/94 


\title{
Postnatal Growth Rates Covary Weakly with Embryonic Development Rates and Do Not Explain Adult Mortality Probability among Songbirds on Four Continents
}

\author{
Thomas E. Martin, ${ }^{1, \star}$ Juan C. Oteyza, ${ }^{2}$ Adam E. Mitchell, ${ }^{2}$ Ahva L. Potticary, ${ }^{2}$ and Penn Lloyd ${ }^{3}$ \\ 1. US Geological Survey, Montana Cooperative Wildlife Research Unit, University of Montana, Missoula, Montana 59812; 2. Montana \\ Cooperative Wildlife Research Unit, University of Montana, Missoula, Montana 59812; 3. Percy FitzPatrick Institute, Department of \\ Science and Technology/National Research Foundation Centre of Excellence, University of Cape Town, Private Bag X3, Rondebosch \\ 7701, South Africa
}

Submitted July 6, 2014; Accepted October 17, 2014; Electronically published January 21, 2015

Online enhancement: appendix. Dryad data: http://dx.doi.org/10.5061/dryad.ks62j.

\begin{abstract}
Growth and development rates may result from genetic programming of intrinsic processes that yield correlated rates between life stages. These intrinsic rates are thought to affect adult mortality probability and longevity. However, if proximate extrinsic factors (e.g., temperature, food) influence development rates differently between stages and yield low covariance between stages, then development rates may not explain adult mortality probability. We examined these issues based on study of 90 songbird species on four continents to capture the diverse life-history strategies observed across geographic space. The length of the embryonic period explained little variation (ca. 13\%) in nestling periods and growth rates among species. This low covariance suggests that the relative importance of intrinsic and extrinsic influences on growth and development rates differs between stages. Consequently, nestling period durations and nestling growth rates were not related to annual adult mortality probability among diverse songbird species within or among sites. The absence of a clear effect of faster growth on adult mortality when examined in an evolutionary framework across species may indicate that species that evolve faster growth also evolve physiological mechanisms for ameliorating costs on adult mortality. Instead, adult mortality rates of species in the wild may be determined more strongly by extrinsic environmental causes.
\end{abstract}

Keywords: life history, adult mortality, nest predation, nestling growth rates, incubation periods.

\section{Introduction}

Growth and development rates are critical elements of lifehistory strategies and theory (Roff 2002). Faster growth and development can arise from various physiological mechanisms, such as faster metabolism (Arendt 1997; West et al. 2001) or trade-offs among developing physiological

\footnotetext{
* Corresponding author; e-mail: tom.martin@umontana.edu.
}

Am. Nat. 2015. Vol. 185, pp. 380-389. (C) 2015 by The University of Chicago. 0003-0147/2015/18503-55601\$15.00. All rights reserved.

DOI: $10.1086 / 679612$ systems (reviewed in Arendt 1997). These mechanisms and trade-offs can create physiological and phenotypic costs that may compromise adult survival and longevity (McCay 1933; Olsson and Shine 2002; Rollo 2002; Metcalfe and Monaghan 2003). Across various taxa, studies within species have provided experimental support for this intrinsic processes hypothesis, where faster growth rates yielded higher adult mortality (e.g., Olsson and Shine 2002; Rollo 2002; Lee et al. 2013). Yet, experimental manipulations of growth rates within species are tests of proximate responses. Species that evolve faster growth rates may also evolve mechanisms for ameliorating physiological costs on mortality and longevity, such as shifts in fatty acid composition of mitochondrial membranes or cellular repair mechanisms (e.g., Hulbert et al. 2007). Such effects could obviate a relationship between growth rates and adult mortality among species. Moreover, adult mortality may be influenced more strongly by environmental influences such as predation, migration, or non-breeding season stressors (e.g., Rowley and Russell 1991; Sillett and Holmes 2002; Evans et al. 2006; Turbill et al. 2011) than by physiological costs of growth and development. Thus, the influence of variation in growth rates on adult mortality among species in the wild is unclear and deserves a broad test because of the implications for life-history theory.

The influence of intrinsic processes on growth and development rates is thought to be genetically based (Arendt 1997; West et al. 2001). As such, growth and development rates of differing life stages might be expected to positively covary when intrinsic processes are the dominant cause of growth and development rates. In other words, species with long embryonic periods might be expected to also exhibit long postnatal periods and slow growth rates. On the other hand, growth and development rates also may be influenced by extrinsic factors, such as temperature or 
food (Arendt 1997; Badyaev and Martin 2000; Gillooly et al. 2002; Martin et al. 2007, 2013; Remeš 2007). Such proximate extrinsic effects may not create the intrinsic costs that can affect adult mortality as expected when development rates are determined primarily by intrinsic processes (Martin and Schwabl 2008; Martin et al. 2013). If proximate extrinsic factors play a strong but differential role between stages, then we might expect low covariance between life stages.

Songbirds provide an interesting system to test these issues. First, lengths of embryonic and nestling periods are thought to be strongly correlated (Moreau and Moreau 1940; Lack 1968; Skutch 1976), potentially reflecting a strong role of intrinsic processes. However, broad phylogenetic tests are lacking. Second, lengths of embryonic periods are negatively correlated with adult mortality probability across species both within and among geographic regions (Martin 2002; Ricklefs 2006; Remeš 2007), which may support the intrinsic processes hypothesis. Yet, this correlation may be an indirect result of adult mortality acting on parental effort to influence embryonic development time (Martin 2002). As a result, the importance of physiological costs from faster growth and development for adult mortality remain unclear, at least in the embryonic stage.

Third, the nestling stage may provide a clearer test of the intrinsic processes hypothesis. Variation in embryonic development rates among species is strongly influenced by the proximate extrinsic effects of temperature (Martin 2002; Martin et al. 2007). Temperature is less important in the nestling period as young develop the ability to thermoregulate, and food delivery does not explain variation in nestling growth and development rates (Martin et al. 2011). As a result, intrinsic effects may be more clearly expressed in the nestling stage, as suggested by correlations between growth rates and metabolic rates (Drent and Klaassen 1989). These differing influences of intrinsic and extrinsic effects between the two stages predict low covariance of development rates between the two stages.

Fourth, increased nest predation risk can exert selection to favor evolution of faster growth rates among diverse species (Remeš and Martin 2002; Martin et al. 2011). This faster growth appears to be achieved in part through physiological trade-offs (Cheng and Martin 2012). Such physiological trade-offs, together with metabolic influences on growth rates (Drent and Klaassen 1989), provide a reasonable basis for the intrinsic processes hypothesis. An initial test among songbird species of North America found that adult mortality probability was weakly related to nestling growth rates but not to length of nestling periods (Remeš 2007). Yet, nestling growth rates and periods in this test were corrected for nest predation effects (Remeš
2007), and nest predation can underlie intrinsic trade-offs (Cheng and Martin 2012) that are thought to influence adult mortality. The low amount of variance explained for growth rates, the inconsistent results between metrics (growth rates vs. nestling period duration), and the removal of potential intrinsic effects due to nest predation suggest that further tests are needed. Moreover, songbirds exhibit much greater variation in these life-history traits when examined across geographic space than within North America alone (Martin et al. 2000, 2007, 2011; Sandercock et al. 2000; Ghalambor and Martin 2001; Lloyd et al. 2014). Thus, comparisons across latitudes are needed to allow examination of potential relationships across relatively large geographic shifts in life-history strategies.

Here, we report tests based on field studies of 90 species on four continents. We examined whether lengths of nestling periods and growth rates covaried with lengths of embryonic periods, to explore whether species-specific intrinsic processes underlie the development rates of these two life stages. We further tested the hypothesis that nest predation exerts selection on nestling periods and growth rates that are potentially achieved through physiological trade-offs that explain variation in adult mortality probability. Finally, we tested the ability of nestling period durations and growth rates to explain variation in adult mortality probability.

\section{Material and Methods}

$$
\text { Study Areas }
$$

We studied 90 passerine species on four continents (fig. A1, available online). We were able to obtain exact observations of embryonic and nestling development times (see below) for these 90 species. We measured growth rates on 80 of these species and were able to estimate annual adult mortality probability for 66 species. The species were studied in north temperate Arizona $\left(34^{\circ} \mathrm{N}\right)$, tropical Venezuela $\left(9^{\circ} \mathrm{N}\right)$, tropical Malaysia $\left(6^{\circ} \mathrm{N}\right)$, and south temperate South Africa $\left(34^{\circ} \mathrm{S}\right)$, representing a broad phylogenetic range of songbirds (fig. A1). Nests were studied in northcentral Arizona for 26 years (1987-2012) and adult mortality for 21 years (1993-2013) at about 2,350-m elevation in mixed deciduous and coniferous forest (Martin et al. 2007). Nests and adult mortality were studied in the tropics for 7 years (2002-2008) in primary forest in Yacambu National Park, Venezuela, at elevations of 1,400-2,000 m (Martin et al. 2007) and for 5 years (2009-2013) in Kinabalu Park, Malaysia, at 1,450-1,950-m elevation (Martin et al. 2013). Nests were studied for 5 years (2000-2004), and adult mortality for 7-8 years (2001-2007), in south temperate coastal dwarf shrubland near Cape Town, South Africa, at sea level (Martin et al. 2007). 


\section{Nest Predation Rates, Development Times, and Nestling Growth Rates}

For species examined here, large numbers of nests were monitored following long-term protocols (Martin et al. $2007,2011)$. The embryonic period was quantified as the number of days between last egg laid and last egg hatched (Martin et al. 2007). The nestling period was quantified as the number of days between the last egg hatched and the last nestling to leave the nest (Martin et al. 2011). Nests were checked every 2-4 days to determine status and predation events but were checked daily or twice daily during egg laying, near hatching, and near fledging to obtain exact period durations. Nest predation was assumed when all nestlings disappeared more than 2 days prior to average fledging age and parents could not be found in the area feeding fledglings.

We weighed nestlings using portable electronic scales with an accuracy of $\pm 0.001 \mathrm{~g}$. We weighed nestlings every day for the first 3 days starting on hatch day and then every other day, or simply every other day beginning on hatch or the day after hatch. We estimated growth rates using the logistic growth curve because growth is typically $\mathrm{S}$ shaped and this approach produces only three parameters that are readily biologically interpretable based on the equation $W(t)=A /\left\{1+e\left[-k *\left(t-t_{\mathrm{i}}\right)\right]\right\}$, where $W(t)$ denotes body mass of a nestling at time $t, A$ is the asymptotic mass that nestlings approach, $t_{\mathrm{i}}$ is the inflection point of the curve, and $k$ is a constant scaling rate of growth (Ricklefs 1968; Remeš and Martin 2002). The growth rate constant, $k$, is a standardized measure of growth rate that is independent of absolute time and size and thus is particularly useful for comparative studies (Ricklefs 1968; Remeš and Martin 2002).

\section{Adult Mortality}

In Arizona, Venezuela, and Malaysia, nets were deployed in stations of 10 or 12 nets as subplots within and across all nest-searching plots. These netting subplots were deployed three times per breeding season, with 20-25 days before subplots were revisited. Nets at a station were deployed for $6 \mathrm{~h}$ starting at dawn. Netting methods for the South Africa site are detailed in Lloyd et al. (2014). All birds that were captured were banded with numbered metal bands and unique combinations of three color bands (two bands per leg), unless it was a recapture. Color bands were used for resighting by nest searchers who visited each nest plot daily or every other day throughout the season. Resighting and recaptures were used in RMARK (Laake 2013) to estimate annual adult survival probabilities (see "Statistical Analyses").
Statistical Analyses

Daily nest predation rates of birds during the nestling period were estimated using the logistic exposure method (Shaffer 2004) based on R v3.0.3 for Windows (R Development Core Team, Vienna). RMARK (Laake 2013) was used to estimate annual adult survival $(\Phi)$ and resighting/ recapture $(p)$ probabilities (White and Burnham 1999; Burnham and Anderson 2002). For each species, models were built with all additive combinations of $\Phi$ and $p$ assumed to be constant, sex specific, or a transient model based on the first year of capture versus all subsequent years. Thus, the global model was $\Phi$ (sex + transient) $p$ (sex + transient). Sex in tropical sites included an unknown category because many species do not exhibit sexual dimorphism and many individuals are not in breeding condition when captured. Parameter estimates were based on averaging across all 16 models based on model weights (Burnham and Anderson 2002) for all species except those in the South Africa site, where we used previous estimates (Lloyd et al. 2014).

We first examined the covariance of embryonic and nestling development times based on the mean estimates for each species using a linear mixed model based on LME4 (Bates et al. 2014). We examined effects of within- and among-site variances using the approach described by van de Pol and Wright (2009) and included site as a random effect in the model. We also tested the same model with nestling growth rate as the dependent variable. We initially included log-transformed mass as a covariate because of potential allometric effects (e.g., Rahn and Ar 1974; Calder 1984), but mass was never a significant influence on these traits, as previously found for songbirds (e.g., Martin et al. 2007, 2011). As a result, we dropped mass from analyses.

We next examined the ability of nestling period length and nestling growth rate to explain variation in adult mortality probability. Again, we initially included body mass as a covariate because both adult mortality and nest predation may be influenced by adult size (Roff 2002; Biancucci and Martin 2010), but mass was not significant and was dropped from analyses. Adult mortality probability was the dependent variable, with nestling period or nestling growth rates as the covariate. We again examined effects of within- and among-site variances using the approach described by van de Pol and Wright (2009) and included site as a random effect in the model.

We followed this analysis with one where we tested the importance of adult and offspring (i.e., nest predation) mortality to variation in nestling period and nestling growth rates. Nestling period or growth rate was the dependent variable in separate analyses with adult mortality probability and nest predation rates as within- and among- 

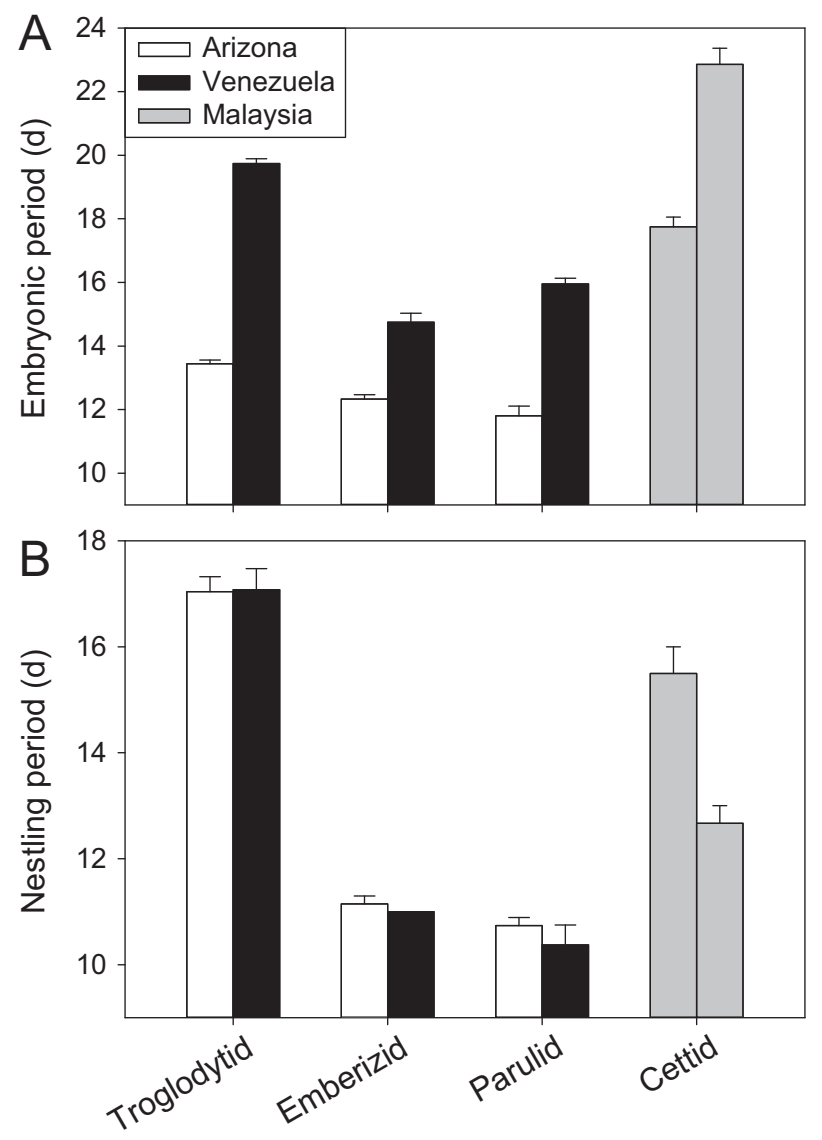

Figure 1: Comparisons of embryonic period (days; $A$ ) and nestling period (days; $B$ ) between related species of three avian families in the north temperate Arizona site versus the tropical Venezuela site and in the Cettid family within the Malaysian Borneo site. Troglodytidae includes Troglodytes aedon in Arizona and Henicorhina leucophrys in Venezuela. Emberizidae includes Junco hyemalis in Arizona and Arremon brunneinucha in Venezuela. Parulidae includes Setophaga virginiae in Arizona and Basileuterus tristriatus in Venezuela. Cettidae includes Cettia vulcania first and Urosphena whiteheadi second, both in Malaysian Borneo.

site covariates using the approach of van de Pol and Wright (2009) and including site as a random factor.

Analyses were first made on raw data. Phylogenetically independent contrasts were also calculated and analyzed to control for possible phylogenetic effects (Felsenstein 1985) using the recent comprehensive phylogeny provided by Jetz et al. (2012). Phylogenetic trees were obtained from http://www.birdtree.org (Jetz et al. 2012) using the Hackett et al. (2008) backbone and imported into Mesquite (Maddison and Maddison 2011) to construct a majority-rule consensus tree based on 500 trees (fig. A1). The trees were time calibrated, such that branch lengths were included in analyses. Contrasts were calculated using the PDAP module (Midford et al. 2002) and imported into IBM SPSS (ver. 22) for linear regressions through the origin. Results using independent contrasts were reported along with analyses of raw data.

\section{Results}

Comparisons of a few pairs of related species provide examples that lengths of embryonic and nestling periods can vary independently of each other (fig. 1). First, comparisons of three pairs of related species between the new world tropics (Venezuela) and north temperate zone (Arizona) show large differences in embryonic periods but no difference in nestling periods between latitudes in three different avian families (fig. 1). Similarly, related species within a site demonstrated the same kinds of divergence between stages. For example, two related species in the family Cettidae in Malaysian Borneo showed large differences in opposing directions between stages; Urosphena
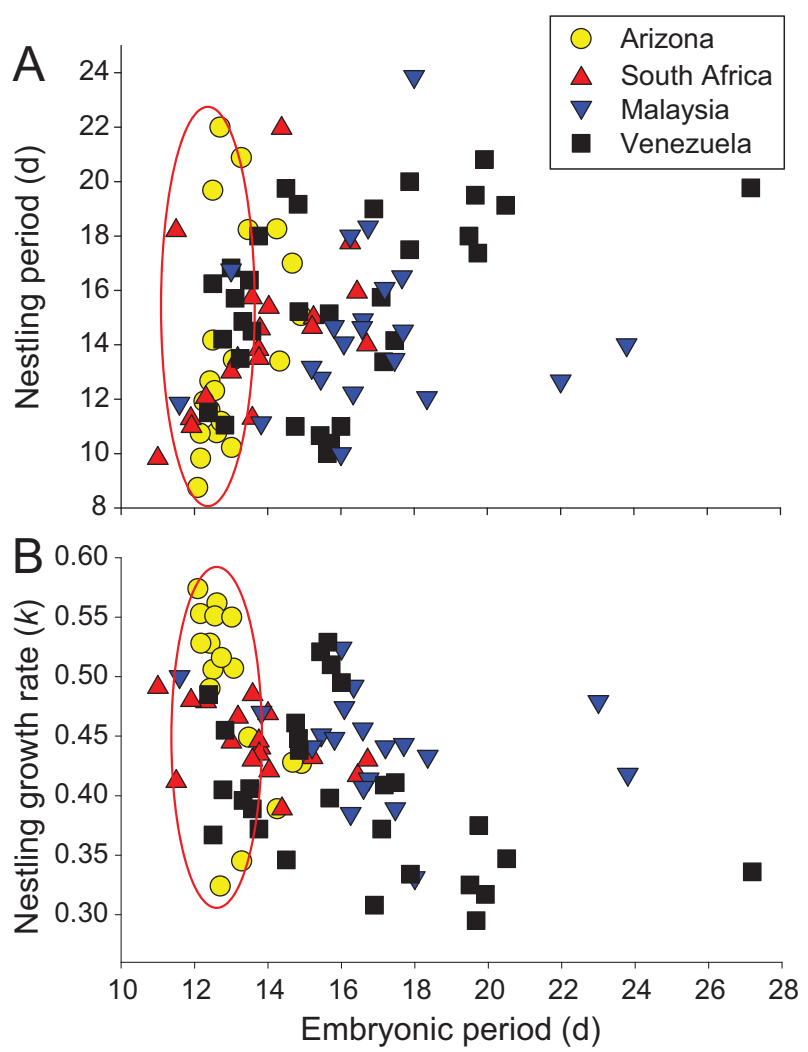

Figure 2: Covariance of nestling period (90 species; $A$ ) and nestling growth rates (80 species; $B$ ) with embryonic development time among four sites spanning north temperate, tropical, and south temperate latitudes. The red ellipses encompass the majority of species from the north temperate Arizona site to demonstrate the narrow range of embryonic development time but large variation in nestling development time and growth rates. 
Table 1: Tests of the covariance of nestling period and nestling growth rate with embryonic period based on mixed-model analyses of raw data with site as a random effect and partitioning withinversus among-site variance and a regression analysis of phylogenetic independent contrasts for songbird species from Arizona, Venezuela, Malaysia, and South Africa

\begin{tabular}{|c|c|c|c|}
\hline Variable & $B(\mathrm{SE})$ & $P$ & $r_{\mathrm{p}}^{2}$ \\
\hline \multicolumn{4}{|l|}{ Mixed model: } \\
\hline \multicolumn{4}{|c|}{ Nestling period (days) as dependent variable ( $n=90$ spp.): } \\
\hline Embryonic period (among sites) & $.208(.238)$ & .45 & $\ldots$ \\
\hline Embryonic period (within sites) & $.477(.139)$ & $<.001$ & $\ldots$ \\
\hline \multicolumn{4}{|c|}{ Nestling growth rate $(k)$ as dependent variable ( $n=80 \mathrm{spp}$.$) :$} \\
\hline Embryonic period (among sites) & $-.012(.004)$ & .004 & $\ldots$ \\
\hline Embryonic period (within sites) & $-.010(.003)$ & .001 & $\ldots$ \\
\hline \multicolumn{4}{|l|}{ Independent contrasts: } \\
\hline \multicolumn{4}{|c|}{ Nestling period (days) as dependent variable ( $n=90 \mathrm{spp}$.$) :$} \\
\hline Site & & .44 & .031 \\
\hline Embryonic period & $.440(.122)$ & .001 & .132 \\
\hline \multicolumn{4}{|c|}{ Nestling growth rate $(k)$ as dependent variable $(n=80 \mathrm{spp}$.$) :$} \\
\hline Site & & .021 & .121 \\
\hline Embryonic period & $-.008(.002)$ & .002 & .126 \\
\hline
\end{tabular}

whiteheadi has a much longer embryonic period but a shorter nestling period compared to Cettia vulcania (fig. 1). Many other similar examples exist. Thus, even related species can exhibit strong independent variation in durations of these two developmental stages.

A more general test among 90 species for the covariance of development time between the two stages indicated that embryonic periods were related to nestling period lengths within sites but not among sites (fig. $2 A$; table 1 ). However, the independent contrast analyses showed that embryonic development time explained only $13 \%$ of the variation in nestling period lengths and nestling periods did not differ among sites (fig. $2 A$; table 1). Indeed, the red ellipse in- cludes most of the Arizona species, which show a relatively narrow variation in duration of the embryonic period but large variation in the nestling period (fig. $2 A$ ), demonstrating that nestling period durations can vary independently of embryonic period durations across species. The lack of significant site effects reflects that variation in nestling period durations in Arizona encompassed the range of variation observed in the other three geographic locations. In contrast, few of the tropical species from Venezuela and Malaysia exhibit embryonic development times within the ellipse representing most of Arizona. Instead, the tropical species generally have longer and more variable embryonic periods than north temperate species.

Table 2: Tests of the ability of nestling period length (days) and nestling growth rate $k$ to predict annual adult mortality probability based on mixed-model analyses of raw data with site as a random effect and partitioning within- versus among-site variance and a regression analysis of phylogenetic independent contrasts for songbird species from Arizona, Venezuela, Malaysia, and South Africa

\begin{tabular}{|c|c|c|c|}
\hline Variable & $B(\mathrm{SE})$ & $P$ & $r_{\mathrm{p}}^{2}$ \\
\hline \multicolumn{4}{|c|}{ Mixed model: } \\
\hline \multicolumn{4}{|c|}{ Adult mortality probability as dependent variable ( $n=66 \mathrm{spp}$.$) :$} \\
\hline Nestling period (among sites) & $-.066(.099)$ & .57 & $\ldots$ \\
\hline Nestling period (within sites) & $.005(.003)$ & .20 & $\ldots$ \\
\hline \multicolumn{4}{|c|}{ Adult mortality probability as dependent variable $(n=62 \mathrm{spp}$.$) :$} \\
\hline Growth rate $k$ (among sites) & $2.432(1.360)$ & .33 & $\ldots$ \\
\hline Growth rate $k$ (within sites) & $-.026(.212)$ & .92 & $\ldots$ \\
\hline \multicolumn{4}{|l|}{ Independent contrasts: } \\
\hline \multicolumn{4}{|c|}{ Adult mortality probability as dependent variable $(n=66 \mathrm{spp}$.$) :$} \\
\hline Site & & $<.001$ & .449 \\
\hline Embryonic period & $.004(.004)$ & .33 & .016 \\
\hline \multicolumn{4}{|c|}{ Adult mortality probability as dependent variable $(n=62 \mathrm{spp}$.$) :$} \\
\hline Site & & $<.001$ & .342 \\
\hline Embryonic period & $.138(.240)$ & .57 & .006 \\
\hline
\end{tabular}



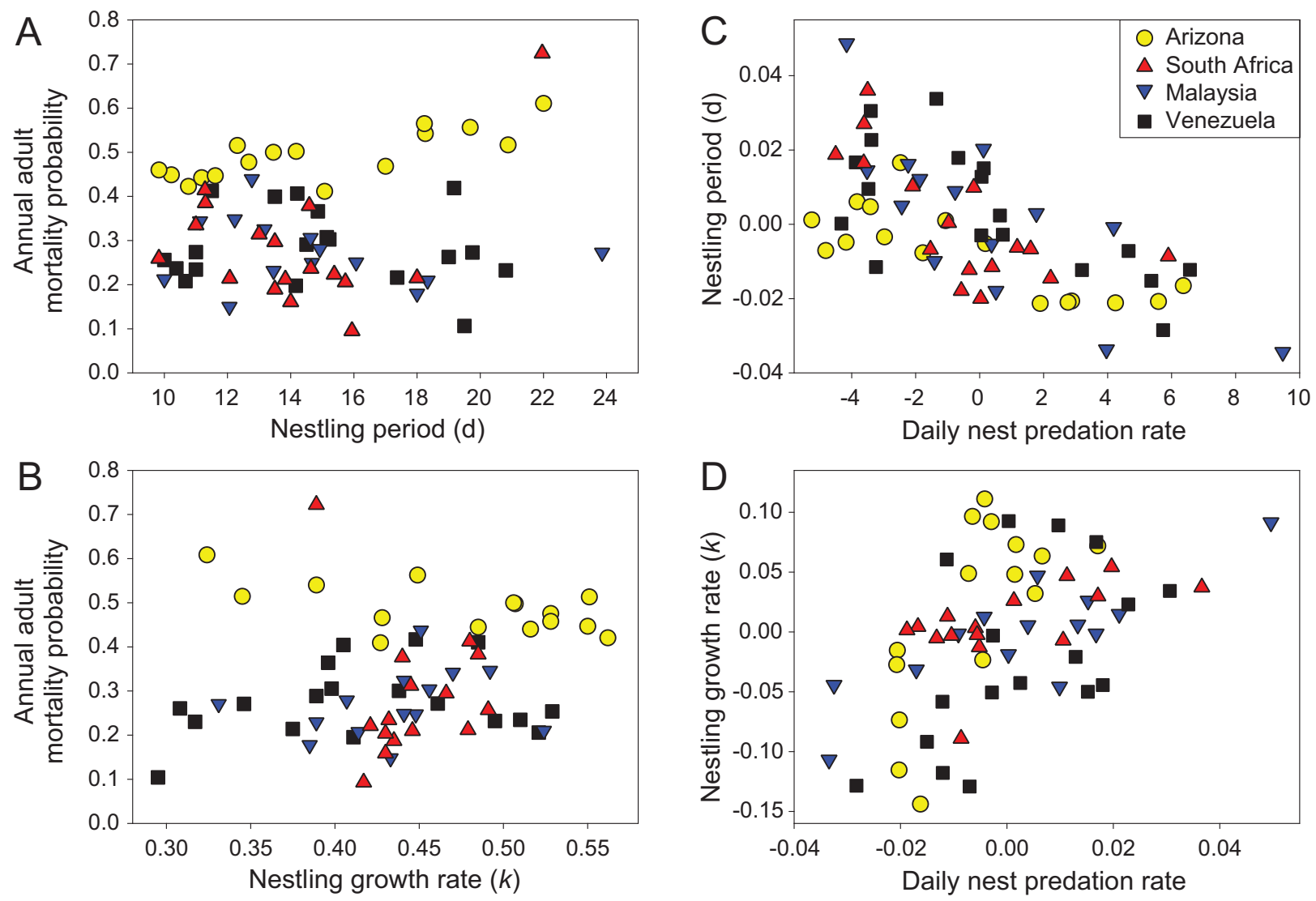

Figure 3: Plots of adult mortality probability as a function of nestling period length (days; $A$ ) and nestling growth rate $(k ; B)$, plus partial regression plots of nestling period length (days; $C$ ) and nestling growth rates $(D)$ as a function of nestling predation rates while accounting for adult mortality probability. We did not correct for site differences to allow illustration of any differences.

These longer embryonic periods were often observed for species that had nestling periods similar to relatives in north temperate Arizona (figs. $1,2 A$ ), demonstrating why embryonic development time explained little variation in nestling development time.

Embryonic development time also was related to nestling growth rate $(k)$ within sites and among sites (fig. $2 B$; table 1). The significant among-sites effect reflects that average growth rates for a site were correlated with average embryonic development times of sites (fig. $2 B$; table 1 ). Nonetheless, embryonic development time still explained only $13 \%$ of the variation in nestling growth rates within and among sites based on the independent contrast analyses (table 1). Indeed, comparisons of temperate species in the red ellipse versus tropical species show similar independence of growth rates between stages as seen for nestling period durations. In particular, tropical species show large differences in embryonic development time from temperate species with similar nestling growth rates (fig. 2B).
A test of the intrinsic processes hypothesis that nestling periods or nestling growth rates explained variation in adult mortality probability across species within and among sites found no hint of support (table 2; fig. 3A, $3 B$ ). The significant site effects in the independent contrast analyses indicated that adult mortality probability differed among sites, being lower on average in tropical sites (table 2). However, this among-site difference was not correlated with nestling development time, as shown by the nonsignificant among-site test in the mixed models (table 2).

A test of the effects of age-specific mortality on nestling period and nestling growth rates similarly showed that adult mortality probability was not important but nest predation was correlated with both measures of nestling development rates (table 3; fig. $3 C, 3 D$ ). The independent contrast analyses showed that nest predation explained $34 \%$ of the variation in nestling period lengths and $27 \%$ of the variation in nestling growth rates (table 3; fig. $3 C$, $3 D)$. 
Table 3: Nestling period length (days) and nestling growth rate $k$ relative to daily nest predation rate during the nestling period and annual adult mortality probability based on mixed-model analyses of raw data with site as a random effect and partitioning within- versus among-site variance and a regression analysis of phylogenetic independent contrasts for songbird species from Arizona, Venezuela, Malaysia, and South Africa

\begin{tabular}{|c|c|c|c|}
\hline Variable & $B(\mathrm{SE})$ & $P$ & $r_{\mathrm{p}}^{2}$ \\
\hline \multicolumn{4}{|l|}{ Mixed model: } \\
\hline \multicolumn{4}{|c|}{ Nestling period as dependent variable ( $n=64$ spp.): } \\
\hline Nest predation rate (among sites) & $-167.7(232.0)$ & .60 & $\ldots$ \\
\hline Nest predation rate (within sites) & $-154.7(18.42)$ & $<.001$ & $\ldots$ \\
\hline Adult mortality (among sites) & $-17.08(23.54)$ & .60 & $\ldots$ \\
\hline Adult mortality (within sites) & $1.932(3.211)$ & .55 & $\ldots$ \\
\hline \multicolumn{4}{|c|}{ Nestling growth rate $k$ as dependent variable ( $n=61 \mathrm{spp}$.): } \\
\hline Nest predation rate (among sites) & $5.116(5.804)$ & .54 & $\ldots$ \\
\hline Nest predation rate (within sites) & $2.427(.392)$ & $<.001$ & $\ldots$ \\
\hline Adult mortality (among sites) & $.715(.590)$ & .44 & $\ldots$ \\
\hline Adult mortality (within sites) & $.052(.067)$ & .44 & $\ldots$ \\
\hline \multicolumn{4}{|c|}{ Independent contrasts: } \\
\hline \multicolumn{4}{|c|}{ Nestling period as dependent variable ( $n=64 \mathrm{spp}$.$) :$} \\
\hline Site & & .010 & .177 \\
\hline Nest predation rate & $-109.2(19.92)$ & $<.001$ & .341 \\
\hline Adult mortality & $.874(3.227)$ & .79 & .001 \\
\hline \multicolumn{4}{|c|}{ Nestling growth rate $k$ as dependent variable ( $n=61 \mathrm{spp}$.$) :$} \\
\hline Site & & .001 & .274 \\
\hline Nest predation rate & $1.856(.418)$ & $<.001$ & .267 \\
\hline Adult mortality & $.089(.069)$ & .17 & .034 \\
\hline
\end{tabular}

\section{Discussion}

The inability of nestling periods and growth rates to explain any variation in adult mortality probability (fig. $3 \mathrm{~A}$, $3 B$; table 2) across a diverse suite of songbird species is counter to long-standing life-history expectations (McCay 1933; Arendt 1997; Metcalfe and Monaghan 2003; Lee et al. 2013). Moreover, this result differs from the relationship between embryonic development time and adult mortality probability observed in passerine species (Martin 2002; Ricklefs 2006; Remeš 2007). These differences between life stages in their correlated variation with adult mortality probability are consistent with the results that nestling development times and growth rates varied largely independently of embryonic development times (figs. 1, 2; table 1).

The independent variation in development rates between stages likely reflects differential effects of extrinsic and intrinsic inputs. The association of adult mortality probability with embryonic development time may result largely through selection on parental effort in warming embryos and influencing development time (Martin 2002). In contrast, temperature is less important to development during the nestling stage of passerine birds because nestlings develop endothermy (Ricklefs 1973; Cheng and Martin 2012). As a result, related species that differ in embryonic periods (fig. $1 A$ ) because of parentally in- duced temperatures (see Martin et al. 2007; Martin and Schwabl 2008) do not necessarily differ in lengths of the nestling period (fig. $1 B$ ). Of course, nestling growth rates may be influenced by other extrinsic influences of parental effort, such as feeding rates. Yet, adult mortality should exert selection on parental effort (Williams 1966; Michod 1979; Reznick et al. 1990; Charlesworth 1994; Ghalambor and Martin 2001), and if parental effort (i.e., feeding rates) has a strong influence on growth rates, then growth rates should be positively correlated with adult mortality. Yet, variation in parental feeding rates did not explain variation in growth rates among diverse species (Martin et al. 2011). Moreover, the absence of any relationships between nestling growth rates and adult mortality (fig. $3 A, 3 B$ ) further suggests that selection by adult mortality on parental effort is of minimal importance to variation in growth rates.

The results here are for a single order of birds (Passeriformes), although it encompasses nearly $50 \%$ of the bird species of the world. The applicability of our results to other taxa is unclear. The covariance of embryonic and postnatal development times and rates has not been tested across other taxa with external ectothermic embryos. Yet, substantial independence of the development rates of embryonic and postnatal stages, as we found for songbirds, also might be expected in other taxa. The extrinsic influence of temperature on embryonic development time is 
well documented across diverse ectothermic taxa (Gillooly et al. 2002), in addition to songbirds (Martin 2002; Martin et al. 2007). Yet, in contrast, postnatal growth rates of these taxa may be more strongly influenced by physiological influences (West et al. 2001). One difference between birds and other taxa with external ectothermic embryos is that birds can more strongly alter the temperature environment of the embryo through parental effort in warming eggs (Martin et al. 2007). In contrast, the embryos of other ectothermic species are often simply left exposed to environmental temperatures of the site where they place eggs. Of course, ectothermic species can alter the temperature environment through nest site selection and even parental care in some species, but such temperature modulation is still limited and can have large effects on embryo development rate (Deeming and Ferguson 1991; Madsen and Shine 1999; Angilletta et al. 2009). In contrast to altricial songbirds, temperature effects on development rate may be reduced for postnatal young of ectothermic species because they have the ability to move and behaviorally regulate temperature. Thus, embryonic and postnatal development times of other taxa may also show large divergences due to differing extrinsic and intrinsic inputs between stages, and these possibilities deserve study.

As thought to be true of diverse taxa (i.e., West et al. 2001), variation in postnatal growth rates of birds may be more strongly influenced by intrinsic processes than extrinsic parental effects compared with the embryonic period (Martin et al. 2007; Cheng and Martin 2012). Faster postnatal growth seems to reflect the role of faster metabolism, a key intrinsic process (Drent and Klaassen 1989; West et al. 2001). Moreover, increased nest predation plays an important role in favoring evolution of faster nestling growth rates among bird species (fig. $3 B$; also Remeš and Martin 2002; Martin et al. 2011) and may underlie physiological trade-offs of birds and other taxa (Arendt 1997; Cheng and Martin 2012). These physiological processes and trade-offs underlying growth rate variation can create the physiological costs that are thought to compromise adult survival and longevity among diverse organisms (McCay 1933; Arendt 1997; Metcalfe and Monaghan 2003; Lee et al. 2013). Yet, the lack of covariance of nestling growth rates with adult mortality suggests that these intrinsic costs are of minor importance to the broader range of variation in adult mortality among wild species of differing geographic regions (fig. $3 A, 3 B$; table 2).

While faster growth rates may incur physiological costs, species that evolve faster growth may also evolve physiological mechanisms to offset these costs and their effects on longevity, such as variation in the fatty acid composition of mitochondrial membranes or cellular repair mechanisms (Hulbert et al. 2007). Indeed, the fact tha $\rightarrow$ flying organisms have greater longevity despite higher met- abolic rates for the same body size as nonflying organisms (Holmes and Austad 1995) demonstrates that physiological costs, such as those produced by metabolism, can be ameliorated. Instead, external sources of mortality imposed by predators, migration, or stressors during reproduction or lean seasons (Rowley and Russell 1991; Sillett and Holmes 2002; Leyrer et al. 2013) may be more important in driving the majority of adult mortality and longevity for species in the wild (also see Reznick et al. 2004).

In conclusion, development times and rates may vary independently between life stages because proximate external influences, such as temperature, can differ between stages and mask underlying physiological programs that might be similar between stages (e.g., Martin et al. 2013). Physiological costs associated with evolved differences in growth rates among species may not manifest in effects on adult mortality and longevity because species may also evolve mechanisms to ameliorate such costs. Instead, external sources of adult mortality may have a more important role in explaining broad variation in adult mortality among diverse species in the wild. Assumptions that growth rates influence adult mortality and longevity need to be viewed with more caution in an evolutionary framework.

\section{Acknowledgments}

We are grateful to R. M. Callaway, R. Ton, and two anonymous reviewers for helpful comments on the manuscript. We are also grateful to M. Lakim, Sabah Parks, and the Sabah Biodiversity Centre for their help in Malaysia; G. Greeff, Eskom, and the Western Cape Nature Conservation Board in South Africa; and C. Bosque, Instituto Nacional de Parques, and Fonacit in Venezuela. This work was supported by the National Science Foundation (DEB0543178, DEB-0841764, DEB-1241041, IOS-1349178) and the US Geological Survey Climate Change Research Program. This work was conducted under University of Montana Institutional Animal Care and Use Committee 05910TMMCWRU. Any use of trade, firm, or product names is for descriptive purposes only and does not imply endorsement by the US government.

\section{Literature Cited}

Angilletta, M. J., Jr., M. W. Sears, and R. M. Pringle. 2009. Spatial dynamics of nesting behavior: lizards shift microhabitats to construct nests with beneficial thermal properties. Ecology 90:29332939.

Arendt, J. D. 1997. Adaptive intrinsic growth rates: an integration across taxa. Quarterly Review of Biology 72:149-177. 
$\rightarrow$ Badyaev, A. V., and T. E. Martin. 2000. Individual variation in growtl $\rightarrow$ trajectories: phenotypic and genetic correlations in ontogeny of the house finch (Carpodacus mexicanus). Journal of Evolutionary Biology 13:290-301.

Bates, D., M. Maechler, B. Bolker, and S. Walker. 2014. lme4: linea $\rightarrow$ mixed-effects models using Eigen and S4. R package. Version 1.17. http://CRAN.R-project.org/package $=1 \mathrm{me} 4$.

$\rightarrow$ Biancucci, L., and T. E. Martin. 2010. Can selection on nest size from nest predation explain the latitudinal gradient in clutch size? Journal of Animal Ecology 79:1086-1092.

Burnham, K. P., and D. R. Anderson. 2002. Model selection ancmultimodel inference: a practical information-theoretic approach. Springer, New York.

Calder, W. A., III. 1984. Size, function and life history. Harvarc $\rightarrow$ University Press, Cambridge, MA.

Charlesworth, B. 1994. Evolution in age-structured populations. Cambridge University Press, Cambridge.

$\rightarrow$ Cheng, Y., and T. E. Martin. 2012. Nest predation risk and growth strategies of passerine species: grow fast or develop traits to escape risk? American Naturalist 180:285-295.

Deeming, D. C., and M. W. J. Ferguson, eds. 1991. Egg incubation its effects on embryonic development in birds and reptiles. Cambridge University Press, Cambridge.

Drent, R., and M. Klaassen. 1989. Energetics of avian growth: the causal link with BMR and metabolic scope. Pages 349-359 in C. Bech and R. E. Reinertsen, eds. Physiology of cold adaptation ir birds. Plenum, New York.

$\rightarrow$ Evans, S. B., L. D. Mech, P. J. White, and G. A. Sargeant. 2006. Survival of adult female elk in Yellowstone following wolf resto ration. Journal of Wildlife Management 70:1372-1378.

$\rightarrow$ Felsenstein, J. 1985. Phylogenies and the comparative method. American Naturalist 125:1-15.

$\rightarrow$ Ghalambor, C. K., and T. E. Martin. 2001. Fecundity-survival tradeoffs and parental risk-taking in birds. Science 292:494-497.

$\rightarrow$ Gillooly, J. F., E. L. Charnov, G. B. West, V. M. Savage, and J. H. Brown. 2002. Effects of size and temperature on developmental time. Nature 417:70-73.

$\rightarrow$ Hackett, S. J., R. T. Kimball, S. Reddy, R. C. K. Bowie, E. L. Braun, M. J. Braun, et al. 2008. A phylogenomic study of birds reveals their evolutionary history. Science 320:1763-1768.

$\rightarrow$ Holmes, D. J., and S. N. Austad. 1995. The evolution of avian senescence patterns: implications for understanding primary aging processes. American Zoologist 35:307-317.

$\rightarrow$ Hulbert, A. J., R. Pamplona, R. Buffenstein, and W. A. Buttemer. 2007. Life and death: metabolic rate, membrane composition, and life span of animals. Physiological Reviews 87:1175-1213.

$\rightarrow$ Jetz, W., G. H. Thomas, J. B. Joy, K. Hartmann, and A. O. Mooers. 2012. The global diversity of birds in space and time. Nature 491 . 444-448.

Laake, J. L. 2013. RMark: an R interface for analysis of capturerecapture data with MARK. Alaska Fisheries Science Center Processed Report 2013-01. National Oceanic and Atmospheric Administration, Alaska Fisheries Science Center, National Marine Fisheries Service, Seattle.

Lack, D. 1968. Ecological adaptations for breeding in birds. Methuen, London.

$\rightarrow$ Lee, W. S., P. Monaghan, and N. B. Metcalfe. 2013. Experimentademonstration of the growth rate-lifespan trade-off. Proceedings of the Royal Society B: Biological Sciences 280:20122370. http:// dx.doi.org/10.1098/rspb.2012.2370.
Leyrer, J., T. Lok, M. Brugge, B. Spaans, B. K. Sandercock, and T. Piersma. 2013. Mortality within the annual cycle: seasonal survival patterns in Afro-Siberian red knots Calidris canutus canutus. Journal of Ornithology 154:933-943. doi:10.1007/s10336-013-0959-y.

Lloyd, P., F. Abadi, R. Altwegg, and T. E. Martin. 2014. South-temperate birds have higher apparent adult survival than tropical birds in Africa. Journal of Avian Biology 45:493-500.

Maddison, W. P., and D. R. Maddison. 2011. Mesquite: a modular system for evolutionary analysis. Version 2.75. http:// mesquiteproject.org.

Madsen, T., and R. Shine. 1999. Life history consequences of nestsite variation in tropical pythons (Liasis fuscus). Ecology 80:989997.

Martin, T. E. 2002. A new view for avian life history evolution tested on an incubation paradox. Proceedings of the Royal Society B: Biological Sciences 269:309-316.

Martin, T. E., S. K. Auer, R. D. Bassar, A. Niklison, and P. Lloyd. 2007. Geographic variation in avian incubation periods and parental influences on embryonic temperature. Evolution 61:25582569.

Martin, T. E., P. Lloyd, C. Bosque, D. Barton, L. Biancucci, Y. Cheng, and R. Ton. 2011. Growth rate variation among passerine species in temperate and tropical sites: an antagonistic interaction between parental food provisioning and nest predation risk. Evolution 65: 1607-1622.

Martin, T. E., P. R. Martin, C. R. Olson, B. J. Heidinger, and J. J. Fontaine. 2000. Parental care and clutch sizes in North and South American birds. Science 287:1482-1485.

Martin, T. E., and H. Schwabl. 2008. Variation in maternal effects and embryonic development rates among passerine species. Philosophical Transactions of the Royal Society B: Biological Sciences 363:1663-1674.

Martin, T. E., R. Ton, and A. Niklison. 2013. Intrinsic vs. extrinsic influences on life history expression: metabolism and parentallyinduced temperature influences on embryo development rate. Ecology Letters 16:738-745.

$\rightarrow$ McCay, C. M. 1933. Is longevity compatible with optimum growth? Science 77:410-411.

$\rightarrow$ Metcalfe, N. B., and P. Monaghan. 2003. Growth versus lifespan: perspectives from evolutionary ecology. Experimental Gerontology 38:935-940.

Michod, R. E. 1979. Evolution of life histories in response to agespecific mortality factors. American Naturalist 113:531-550.

Midford, P. E., T. Garland Jr., and W. Maddison. 2002. PDAP: PDTREE package for Mesquite. Version 1.00. http://www. mesquiteproject.org/pdap_mesquite/.

Moreau, R. E., and W. M. Moreau. 1940. Incubation and fledging periods of African birds. Auk 57:313-325.

$\rightarrow$ Olsson, M., and R. Shine. 2002. Growth to death in lizards. Evolution 56:1867-1870.

Rahn, H., and A. Ar. 1974. The avian egg: incubation time and water loss. Condor 76:147-152.

$\rightarrow$ Remeš, V. 2007. Avian growth and development rates and age-specific mortality: the roles of nest predation and adult mortality. Journal of Evolutionary Biology 20:320-325.

Remeš, V., and T. E. Martin. 2002. Environmental influences on the evolution of growth and developmental rates in passerines. Evolution 56:2505-2518.

$\rightarrow$ Reznick, D. A., H. Bryga, and J. A. Endler. 1990. Experimentally 
induced life-history evolution in a natural population. Nature $346 \rightarrow$ Shaffer, T. L. 2004. A unified approach to analyzing nest success. Auk 357-359. 121:526-540.

$\rightarrow$ Reznick, D. N., M. J. Bryant, D. Roff, C. K. Ghalambor, and D. E. $\rightarrow$ Ghalambor. 2004. Effect of extrinsic mortality on the evolution of senescence in guppies. Nature 431:1095-1099.

$\rightarrow$ Ricklefs, R. E. 1968. Patterns of growth in birds. Ibis 110:419-451. . 1973. Patterns of growth in birds. II. Growth rate and mode of development. Ibis 115:117-201.

$\rightarrow-$ 2006. Embryo development and ageing in birds and mammals. Proceedings of the Royal Society B: Biological Sciences 273: 2077-2082.

Roff, D. A. 2002. Life history evolution. Sinauer Associates, Sunderland, MA.

Sillett, T. S., and R. T. Holmes. 2002. Variation in survivorship of a migratory songbird throughout its annual cycle. Journal of Animal Ecology 71:296-308.

Skutch, A. F. 1976. Parent birds and their young. University of Texas Press, Austin.

$\rightarrow$ Turbill, C., C. Bieber, and T. Ruf. 2011. Hibernation is associated with increased survival and the evolution of slow life histories among mammals. Proceedings of the Royal Society B: Biological Sciences 278:3355-3363.

$\rightarrow$ van de Pol, M. V., and J. Wright. 2009. A simple method for distinguishing within- versus between-subject effects using mixed models. Animal Behavior 77:753-758

$\rightarrow$ Rollo, C. D. 2002. Growth negatively impacts the life span of mam $\rightarrow$ West, G. B., J. H. Brown, and B. J. Enquist. 2001. A general model mals. Evolution and Development 4:55-61. for ontogenetic growth. Nature 413:628-631.

Rowley, I., and E. Russell. 1991. Demography of passerines in th $\rightarrow$ White, G. C., and K. P. Burnham. 1999. Program MARK: survival temperate southern hemisphere. Pages $22-44$ in C. M. Perrins, J.D. Lebreton, and G. J. M. Hirons, eds. Bird population studies: relevance to conservation and management. Oxford University Press, New York.

$\rightarrow$ Sandercock, B. K., S. R. Beissinger, S. H. Stoleson, R. R. Melland, and C. R. Hughes. 2000. Survival rates of a Neotropical parrot: implications for latitudinal comparisons of avian demography. Ecology 81:1351-1370.

Associate Editor: Edmund D. Brodie III Editor: Judith L. Bronstein
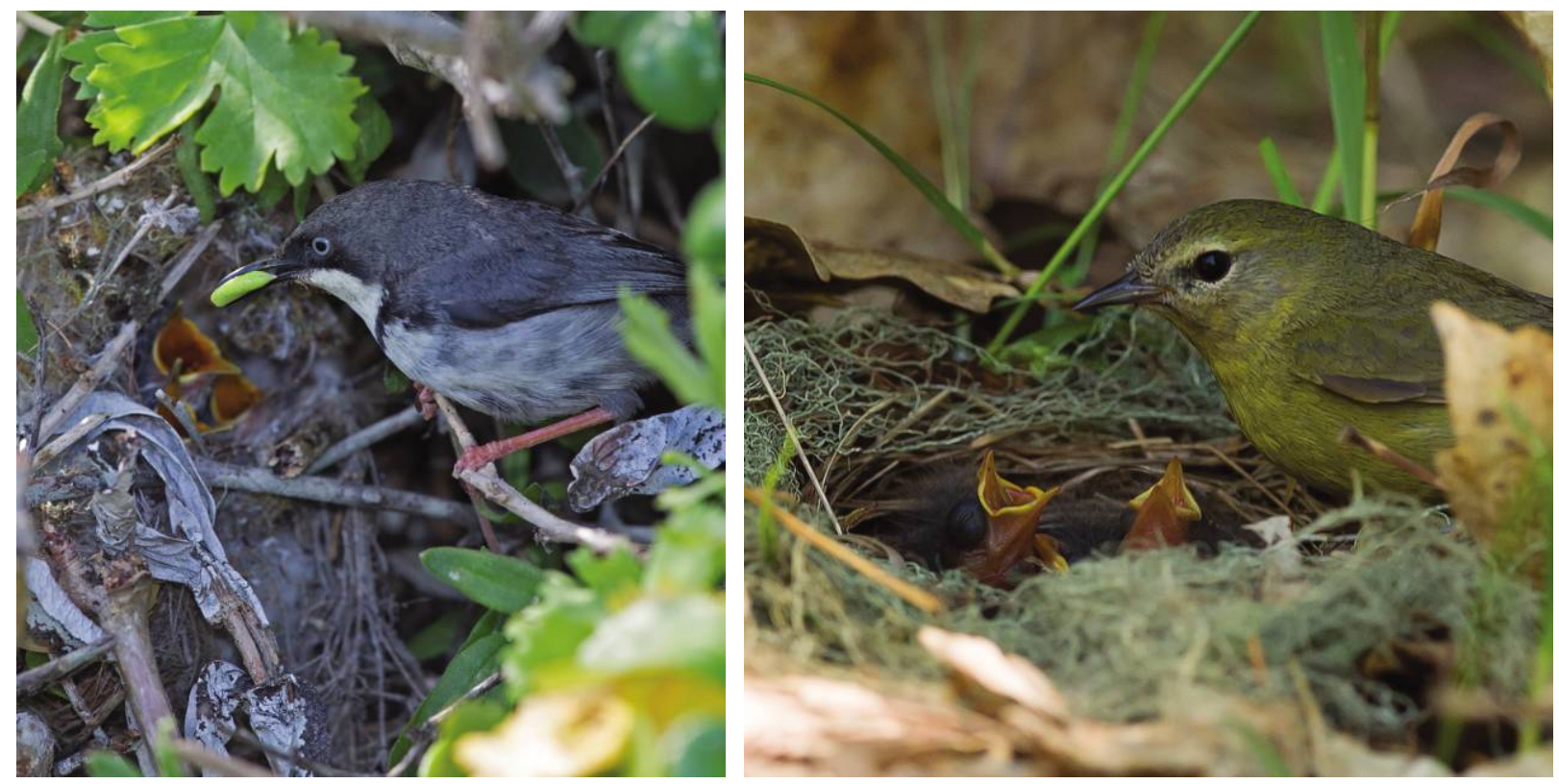

Left, Apalis thoracica feeding young at its nest in the South Africa site. This small (11-g) bird can live more than 15 years. Right, these blind and relatively naked 3-day-old nestlings of the warbler Oreothlypis celata from the Arizona site will grow fast enough to open their eyes and leave the nest fully feathered in 7-8 more days. Young of related tropical warblers in the Venezuela site leave the nest after the same number of days, despite much higher adult survival rates. Photo credits: T. E. Martin. 